\title{
Ensayos de quimiosensibilidad en cultivos primarios de tumores cerebrales
}

\author{
J.L. Gil-Salú; J. Bosco-López*; M. Domínguez-Villar*; I. Domínguez-Pascual; J. Pérez-Requena**; M.J. Palomo** \\ y M. López-Escobar
}

Servicios de Neurocirugía, Unidad Investigación y **Anatomía Patológica. Hospital Universitario Puerta del Mar. Cádiz. *Medicina Interna y Unidad Investigación. Hospital Clínico de Puerto Real. Cádiz.

\section{Resumen}

Durante los últimos 50 años la quimioterapia (QT) ha jugado un importante papel en el tratamiento del cáncer. Sin embargo, el éxito o fracaso de nuevas drogas para un determinado tipo de cáncer es difícil de predecir. La quimiosensibilidad in vitro es un método atractivo para conocer $a$ priori si ese tumor responderá a una pauta de QT y para determinar la dosis óptima de tratamiento en los enfermos con cáncer.

Objetivo. Conocer la sensibilidad de tumores cerebrales frente a determinados fármacos antineoplásicos.

Material y métodos. Se ensayaron 5 fármacos diferentes (carmustina, camptotecina, taxol, hidroxiurea y tamoxifeno) en los cultivos primarios obtenidos de 7 pacientes con glioblastoma multiforme, 15 pacientes con meningiomas y un paciente con meduloblastoma. Para estudiar la quimiosensibilidad se empleó el test del MTT, midiendo la densidad óptica por espectofotometría a $450 \mathrm{~nm}$.

Resultados. Un total de 49 mediciones fueron realizadas, obteniendo 44 curvas dosis-respuesta válidas. Se emplearon concentraciones desde 10-2 $M$ hasta 10-12 $M$ para cada fármaco ensayado, obteniendo IC50 en cada caso como valor representativo de la sensibilidad del tumor a la droga.

Conclusiones. El test MTT se muestra válido para medir la quimiosensibilidad in vitro de tumores cerebrales a nuevos fármacos.

PALABRAS CLAVE: Tumores cerebrales. Cultivos celulares. Quimiosensibilidad. Test MTT.

Chemosensitivity test on brain tumors

Summary

During last 50 years chemotherapy has played a very important part in the cancer treatment. Howe-

Recibido;6-06-06. Aceptado: 25-08-06 ver, succes or failures of news drugs in one particular cancer its difficult to predict. In vitro chemosensitivity is an attractive method for knowing about responses of a tumor to ChT treatment and assess the best dose in the patient with cancer.

Objective. To know brain tumors sensitivity against antineoplastic drugs.

Methods. Five differents drugs (carmustin, camptotecin, taxol, hydroxyurea and tamoxifen) were tested on short-term cultures from 7 patients with Glioblastoma multiforme, 15 patients with meningiomas and one patient with meduloblastoma. For testing chemosensitivity we used MTT assay, and we measured optic density by spectophotometry to $450 \mathrm{~nm}$.

Results. A total of $\mathbf{4 9}$ measurement were done, getting $\mathbf{4 4}$ valids dose-result curves. For each drug we used from 10-2 $M$ to 10-12 $M$ gap, and IC50 result was representative of tumor sensitivity to the drug.

Conclusion. our data support MTT assays like valid method for measuring in vitro chemosensitivity in brain tumors to news drugs.

KEY WORDS: Brain tumors. Cell culture. Chemosensitivity. MTT assay.

Introducción

El empleo de fármacos antineoplásicos es un método de tratamiento, principal o complementario, ampliamente aceptado en muchos tumores cerebrales. Como agentes citotóxicos sirven para prolongar la supervivencia de los pacientes en determinados tipos tumorales. Sin embargo, otros tumores se muestran quimiorresistentes, por ejemplo el glioblastoma multiforme (GM), o es interesante valorar en ellos el papel citorreductor de nuevas drogas, por ejemplo en los meningiomas.

La elección última de la quimioterapia a emplear en el paciente que sufre de un tumor cerebral depende de múltiples factores: la sensibilidad de las células del tumor a las drogas testadas in vitro o in vivo; la penetrabilidad del fármaco en el sistema nervioso central, atravesando la 
barrera hematoencefálica; el índice terapéutico o relación entre la eficacia/toxicidad a las concentraciones empleadas y la farmacocinética del agente citotóxico ${ }^{12}$.

El primero de estos factores está condicionado por las características genéticas del tumor ${ }^{9} \mathrm{y}$, de las mismas, depende la quimiosensibilidad o quimiorresistencia que presente cuando lo valoremos in vitro (en medios de cultivo) o in vivo (modelos animales, valoración clínicoradiológica en pacientes) ${ }^{16}$.

Los métodos que se emplean inicialmente para descubrir la utilidad, y el posterior desarrollo, de un fármaco antineoplásico emplean los cultivos celulares y después los modelos animales en roedores como banco de pruebas. Frente a los cultivos de células tumorales obtenidos por múltiples pases y selección de clones, con una características muy definidas, los cultivos primarios aportan la ventaja de mostrarnos una población de células tumorales heterogénea y ser representativos del paciente a $\operatorname{tratar}^{1,8,20}$.

Con el propósito de valorar la utilidad que los cultivos primarios de tumores cerebrales tienen como banco de pruebas, hemos demostrado anteriormente que son representativos del tumor del que proceden, reproduciendo in vitro las características citológicas e inmunohistoquímicas que en la parafina se demuestran ${ }^{10}$. Conseguido esto, nos proponemos analizar si el test del MTT (3-4,5 dimethylthiazol-2,5-diphenyltetrazolium bromide thiazol blue) es válido para mostrar si hay respuesta a un determinado quimioterápico y a qué concentraciones se obtienen las inhibiciones del crecimiento celular tumoral al 50\% (IC50) en cultivos primarios de tumores cerebrales.

\section{Material y método}

\section{Preparación del cultivo primario}

La obtención del cultivo primario se inicia en el quirófano, con una adecuada toma de la muestra tumoral, unos 4 cc., intentando no fragmentarla, y tomándola de una zona que creamos sea representativa del tumor, evitando por ejemplo áreas de necrosis. Su procesado inmediato lo llevamos a cabo en la Unidad de Investigación de nuestro centro donde, manipulando siempre las muestras en campanas de flujo laminar, se procedía a trocear la muestra, sometiéndola a una digestión enzimática con colagenasa tipo I-a , a $37^{\circ} \mathrm{C}$ durante 90 minutos, en un frasco de Roux con 50 cc de medio de cultivo de Dubelco (DMEM). Posteriormente recogíamos el sobrenadante que centrifugamos a $1600 \mathrm{rpm}$ a $4^{\circ} \mathrm{C}$ durante 7 minutos. El "pellet" o producto de células resultantes era recogido empleando 5 cc de medio de cultivo completo (MCC) (para 5cc: 4.25 cc de DMEM; 50 microlitros de glutamina; 600 microlitros de suero de ternera fetal; 100 microlitros de una solución antibiótica: cloxaciclina, gentamicina y ampicilina), depositándola en un frasco de cultivo que se conservaba en posición hori-

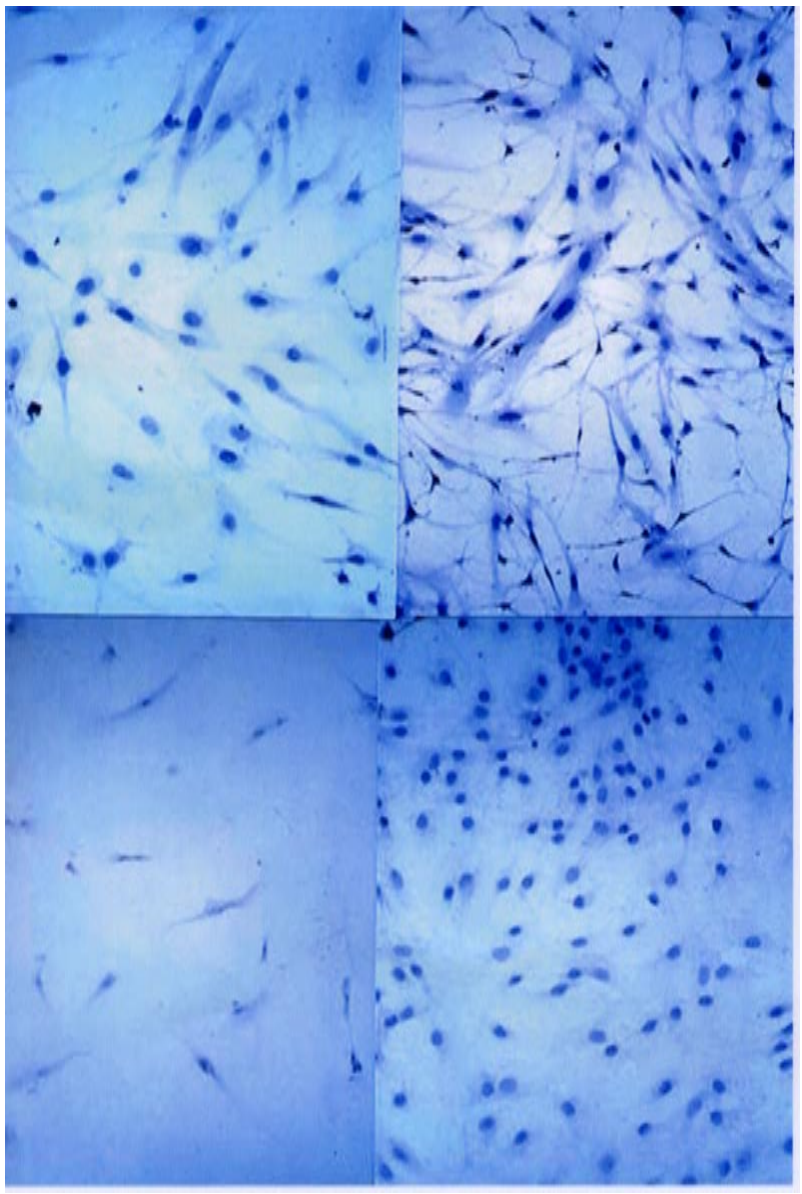

Figura 1. Cultivos primarios de distintos tumores cerebrales en monocapa.

zontal a $37^{\circ} \mathrm{C}$ en un ambiente con $5 \%$ de $\mathrm{CO}_{2}$ durante los días siguientes. En el microscopio de contraste de fases se vigilaba diariamente el crecimiento de las células en cultivo, renovándose cada 4 días el MCC hasta observar el tapizamiento de la superficie del frasco por las células en cultivo en monocapa (Figura 1). En ese momento se procedía a "levantar" las células de la superficie del frasco empleando tripsina, 5 minutos a $37^{\circ} \mathrm{C}$ en ambiente con 5\% de $\mathrm{CO}_{2}$, y luego se neutraliza con suero de ternera fetal, en cantidades equivalentes, y se centrifuga a $1600 \mathrm{rpm}$ a $4^{\circ} \mathrm{C}$ durante 7 minutos, hasta obtener un "pellet" de las células en suspensión ${ }^{10}$.

\section{Realización del test de MTT}

El "pellet" de células es suspendido con DMEM y con la nueva suspensión se llenan los pocillos de una cámara de 96, en la que despreciamos las filas y columnas de los extremos, asignando a cada columna una concentración progresivamente creciente del fármaco a testar (desde 10-2 hasta 10-12 M). Dejamos la cámara en estufa hasta observar el nuevo tapizamiento del fondo (Figura 2). 


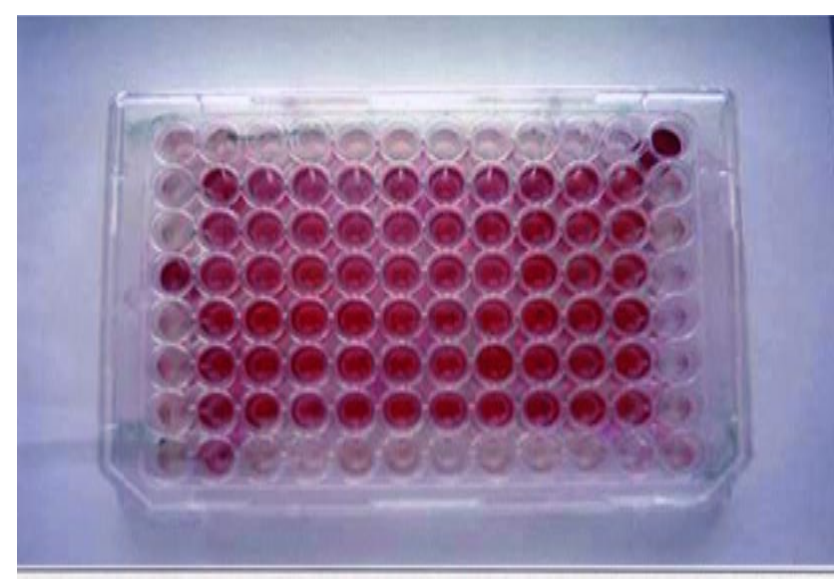

Figura 2. Cámara de 96 pocillos para el MTT test

A continuación incubamos las células de los pocillos con MTT, unos $5 \mathrm{microgr} / \mathrm{ml}$., a $37^{\circ} \mathrm{C}$ durante 4 horas. Se aspira el medio y los cristales de formazan se solubilizan con $60 \mathrm{mM} \mathrm{Hcl}$ en isopropanol, monitorizando la absorvancia a $450 \mathrm{~nm}$ en un espectofotometro/colorímetro. De esta manera se valora la viabilidad celular y al añadir concentraciones progresivamente crecientes del fármaco (se recomienda iniciar las experiencias con concentraciones de entre 10-2 hasta 10-12 Molar), se pueden extrapolar las medias de los resultados a un eje de coordenadas, obteniendo una curva dosis-respuesta, donde podremos calcular la IC50. Este método se basa en la incorporación del MTT a la mitocondria de las células que hayan permanecido o sean viables, que transforman el MTT en cristales de formazan, dotando al medio de coloración más o menos intensa, la cual es valorada por el espectofotómetro. De esta forma, no calculamos el número de células viables, ni el mecanismo de muerte celular implicado, sólo podemos establecer cúal es la IC50 para ese fármaco de ese cultivo primario.

\section{Fármacos a analizar}

Se analizaron 5 fármacos correspondientes a 5 familias distintas de quimioterápicos, algunos de uso habitual en determinados tumores, donde ya han mostrado su eficacia $^{4,17,18}$, todos ellos con un margen de concentración de 10-2 hasta 10-12 M.

- Carmustina: es una nitrosourea, dentro de los agentes alquilantes, que actúa dañando el DNA por carbametilación de los grupos amino.

- Camptotecina: es un inhibidor de la topoisomerasa-I, actuando sobre el huso mitótico durante la duplicación celular.

- Taxol: es un agente que estabiliza los microtúbulos del huso mitótico, inhibiendo la mitosis.

- Hydroxiurea: es un inhibidor específico de una importante enzima, la ribonucleótido difosfatasa reductasa, de la síntesis del DNA.

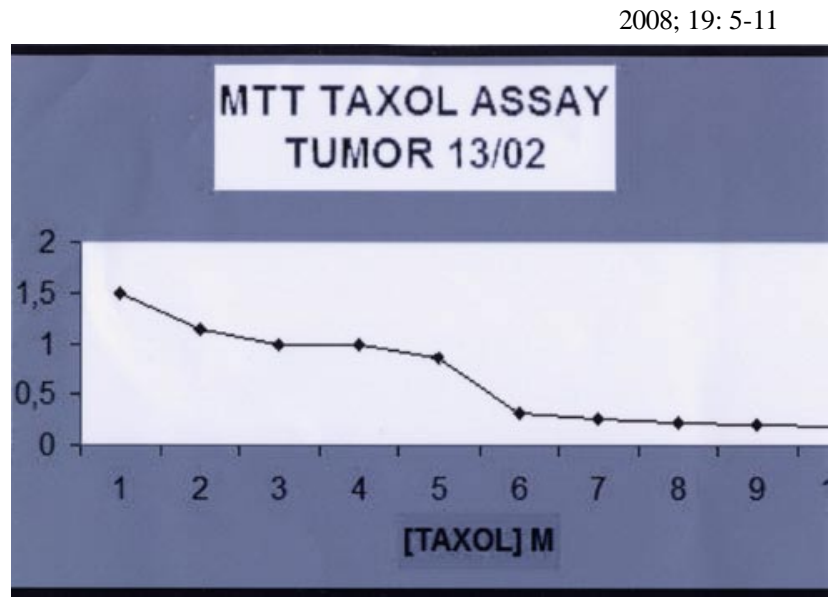

Figura 3. Tipo de curva ejemplo dosis-respuesta.

- Tamoxifeno: es un inhibidor de la protein-kinasa C.

\section{Análisis del test MTT}

La obtención de la lectura en el espectofotómetro nos aporta un valor cuya media, resultante de la lectura de los pocillos que ocupan una columna o, lo que es lo mismo, una concentración del fármaco, nos permitirá elaborar una curva dosis-respuesta (Figura 3). De cada curva obtenemos una IC50 que es el valor que analizamos para cada tumor y fármaco testado.

\section{Resultados}

Los resultados, IC50, obtenidos en el test MU fueron realizados a partir de los cultivos primarios obtenidos de 23 pacientes (7 glioblastoma multiforme (GM), 15 meningiomas (Mng) y 1 meduloblastoma); se llevaron a cabo 49 experiencias, resultando en 5 ocasiones no válida la curva dosis-respuesta obtenida, por lo que analizan un total de 44 curvas y sus correspondientes IC50, que se dividen, como indican las Tablas 1, 2, 3, 4 y 5, según los fármacos testados en los tumores que se señalan.

\begin{tabular}{|c|c|c|}
\hline \multicolumn{3}{c}{ Tabla 1 } \\
\multicolumn{3}{|c|}{ IC-50 para Carmustina (BCNU) } \\
\hline Pacientes & Histopatología & IC-50 (M) \\
\hline 1 & G.M. & $10-6$ \\
\hline 2 & G.M. & $10-8$ \\
\hline 3 & G.M. & $10-5$ \\
\hline 4 & Meningioma & $10-8$ \\
\hline 5 & Meningioma & $10-8$ \\
\hline 6 & Meningioma & $10-4$ \\
\hline 7 & Meningioma & $10-7$ \\
\hline
\end{tabular}

M = molar; G.M. = Glioblastoma multiforme. 
Tabla 2

IC-50 para Camptotecina

\begin{tabular}{|c|c|c|}
\hline Pacientes & Histopatología & IC-50 (M) \\
\hline 2 & G.M. & $10-4$ \\
\hline 6 & G.M. & $10-5$ \\
\hline 8 & G.M. & $10-5$ \\
\hline 12 & G.M. & $10-5$ \\
\hline 15 & G.M. & $10-4$ \\
\hline 3 & Meningioma & $10-7$ \\
\hline 4 & Meningioma & $10-7$ \\
\hline 5 & Meningioma & $10-8$ \\
\hline 9 & Meningioma & $10-5$ \\
\hline 10 & Meningioma & $10-4$ \\
\hline 11 & Meningioma & $10-4$ \\
\hline 13 & Meningioma & $10-5$ \\
\hline 14 & Meningioma & $10-7$ \\
\hline 16 & Meningioma & $10-4$ \\
\hline
\end{tabular}

M = molar; G.M. = Glioblastoma multiforme.

Tabla 3

\section{IC-50 para Taxol}

\begin{tabular}{|l|c|c|}
\hline Pacientes & Histopatología & IC-50 (M) \\
\hline 19 & Meduloblastoma & $10-5$ \\
\hline 17 & Meningioma & $10-5$ \\
\hline 18 & Meningioma & $10-10$ \\
\hline 19 & Meningioma & $10-5$ \\
\hline 20 & Meningioma & $10-7$ \\
\hline
\end{tabular}

$\mathrm{M}=$ molar

Las medias de las IC50 para cada grupo resultaron:

- Carmustina: total 7 curvas
- 3 G.M
IC50 $=10-6$
- 4 Mng IC50 $=10-6$

- Camptotecina: total 14 curvas

- 5 G.M IC50 $=10-4$

- 9 Mng IC50 $=10-5$

- Taxol: total 5 curvas

- 1 meduloblastoma ............. IC50 $=10-5$

• 4 Mng ................................ IC50 = 10-6

- Hidroxiurea: total 10 curvas

- 2 G.M IC50 $=10-4$

- 8 Mng IC50 $=10-4$

- Tamoxifeno: total 8 curvas

- 1 G.M IC50 $=10-6$

- 7 Mng IC50 $=10-7$
Tabla 4

IC-50 para Hidroxiurea

\begin{tabular}{|c|c|c|}
\hline Pacientes & Histopatología & IC-50 (M) \\
\hline 8 & G.M. & $10-5$ \\
\hline 15 & G.M. & $10-4$ \\
\hline 7 & Meningioma & $10-7$ \\
\hline 10 & Meningioma & $10-6$ \\
\hline 11 & Meningioma & $10-6$ \\
\hline 13 & Meningioma & $10-3$ \\
\hline 16 & Meningioma & $10-3$ \\
\hline 20 & Meningioma & $10-5$ \\
\hline 21 & Meningioma & $10-4$ \\
\hline 22 & Meningioma & $10-3$ \\
\hline
\end{tabular}

$\mathrm{M}=$ molar; G.M. = Glioblastoma multiforme.

Tabla 5

IC-50 para Tamoxifeno

\begin{tabular}{|c|c|c|}
\hline Pacientes & Histopatología & IC-50 (M) \\
\hline 8 & G.M. & $10-6$ \\
\hline 3 & Meningioma & $10-10$ \\
\hline 9 & Meningioma & $10-8$ \\
\hline 11 & Meningioma & $10-6$ \\
\hline 14 & Meningioma & $10-9$ \\
\hline 16 & Meningioma & $10-7$ \\
\hline 17 & Meningioma & $10-4$ \\
\hline 23 & Meningioma & $10-6$ \\
\hline
\end{tabular}

$\mathrm{M}=$ molar; G.M. = Glioblastoma multiforme.

\section{Discusión}

Los ensayos de sensibilidad a drogas in vitro son capaces de predecir la respuesta clínica a los agentes quimioterápicos (QMT) en un 50-70\% de los pacientes y de predecir la resistencia clínica en casi el 100\%. Estos ensayos dependen de las características del cultivo celular y no simulan de forma completa las condiciones in vivo. Hay 3 técnicas en el cultivo de los tumores cerebrales para estudiar la quimiosensibilidad: el cultivo en monocapa, el cultivo de órgano y las técnicas de esferoides tumorales multicelulares ${ }^{21}$. Los criterios para medir la sensibilidad pueden expresarse de diversas formas, pero lo más corriente es expresar la concentración de la droga que resulta en un $50 \%$ de reducción de la colonia (IC50). La curva dosis-respuesta obtenida puede ser del tipo 1: tumor 
completamente resistente a la droga ensayada; tipo 2: respuesta inicial, pero se alcanza un nivel estable que expresa la subpoblación de células resistentes; tipo 3: sensibilidad a partir de un umbral inicial a bajas concentraciones y tipo 4: respuesta similar al tipo 3 pero sin el "hombro" inicial (Figura 3$)^{12}$. En nuestras experiencias, la mayor parte de las curvas corresponden al tipo 4, de morfología sigmoidea.

Las limitaciones de este tipo de método experimental pueden ser debidas a 2 factores:

- Intrínsecos: dependientes de las características genotípicas del tumor, de lo que depende su sensibilidad y/o resistencia.

- Extrínsecos: por no simular las características del medio o ambiente propias en las que se desenvuelve el tumor.

Los factores intrínsecos más importantes derivan de la presencia de genes oncosupresores (GOS) y/o oncogenes que median en la respuesta a los fármacos. El ejemplo mejor conocido deriva de la serie que en 1994 Cairncross ${ }^{5}$ publicó sobre los resultados de un ensayo en fase II en 24 pacientes con oligodendrogliomas anaplásicos y oligoastrocitomas tratados con PCV (procarbazina, CCNU y vincristina), obteniendo un $75 \%$ de respuestas (38\% de remisiones completas) según los criterios radiológicos de MacDonald ${ }^{13}$. La alta quimiosensibilidad de esta variante histológica se debe a un déficit de alquil-guanina transferasa, que se encarga de reparar en DNA lesionado por los agentes alquilantes. Los mismos autores evidenciaron que la pérdida del cromosoma lp se correlacionaba en el 100\% de los casos con respuestas objetivas al PCV, con una probabilidad de supervivencia a los 5 años del 95\%. Por otro lado, la resistencia tumoral a los quimioterápicos se ha conocido en los últimos años, con una eclosión en nuestro conocimiento acerca de los mecanismos por los que las células cancerosas se hacen quimiorresistentes ${ }^{2}$. Durante el curso de su evolución (resistencia intrínseca) o en respuesta a la QMT (resistencia adquirida), estas células pueden seguir un número de alteraciones genéticas que les proporcionan un fenotipo resistente común (multidrogas) o específico (individual) ${ }^{19}$. Las aberraciones genómicas, la disregulación de las proteínas transportadoras de membrana y enzimas celulares y la susceptibilidad alterada a entrar en apoptosis están entre las formas que contribuyen a la génesis del fallo en el tratamiento quimioterápico ${ }^{3}$.

Respecto a los factores extrínsecos y en un intento de simular in vitro el crecimiento en tres dimensiones de los tumores objeto de estudio, se han desarrollado métodos de estudio mediante videomicroscopía que permiten valorar la locomoción y proliferación de las células in vitro, las cuales son específicas en cuanto a las características para cada tipo de célula/tumor, manteniéndose en el tiempo éstas a pesar de los distintos "pasos" o subcultivos que se vayan realizando ${ }^{11}$. Para valorar la migración de las células y si se induce o no apoptosis ante diversos tratamientos la motilidad se mide en ensayos de migración en cultivos en monocapa. Por ejemplo se mide como los inhibidores de la tromboxano sintetasa, por medio de la activación de las caspasas (6 horas después del tratamiento) y la consecuente fragmentación del DNA (24-48 horas tras el tratamiento), inducen la apoptosis celular y disminuyen la motilidad celular, e incluso se incrementa la susceptibilidad celular a la fragmentación del DNA tras los tratamientos con camptotecina, etopósido, nitrosoureas y anti-CD95 ${ }^{22}$.

El empleo de sistemas de cultivo mediante matricesgeles de colágeno especiales se ha empleado para ensayar la quimiosensibilidad in vitro de tumores obtenidos de pacientes con glioblastoma y meduloblastoma. Cuando la mayor proporción de las curvas dosis/inhibición se encontraba en el lado de las dosis más bajas, izquierda de las curvas, es decir era igual o mayor al 50\%, el tumor se consideraba sensible al quimioterápico. Medidos de esta forma, los grados de eficacia fueron del 25\% para ACNU, 67\% para adriamicina, 31\% para cisplatino y $68 \%$ para etopósido empleando las matrices de colágeno ${ }^{23}$. La expresión de la PAGF en los cultivos parece relacionarse con el comportamiento del tumor. Así los tumores que muestran menos expresividad a la PAGF son los que se expresan como más agresivos, menos inhibición de contacto y mayor potencial migratorio o invasivo ${ }^{15}$.

Es problemático valorar la capacidad de invasión de los gliomas en cultivo. Por ello, autores como de Boüard et al, demuestran cómo las secciones de cerebro de ratón donde se inocula el cultivo celular tumoral, pueden servir para ello, e intenta cuantificarla, reproduciendo las condiciones in vivo de los cultivos primarios de gliomas o de líneas celulares ${ }^{6,7}$. También se aislan células endoteliales en cultivo a partir de gliomas de diferentes grados para ensayar en ellos fármacos que frenen el crecimiento microvascular tumoral ${ }^{14}$.

Aún asumiendo estas limitaciones del cultivo en monocapa como banco de pruebas para probar la quimiosensibilidad, los resultados por nosotros obtenidos sobre cultivos primarios de G.M., meningiomas y un meduloblastoma, evidencian que es un primer método muy práctico para conocer la utilidad de un nuevo agente citotóxico sobre alguno de estos tipos tumorales, mostrándose los meningiomas más sensibles, con más baja IC50, a los QT que los G.M., así como existe en general mayor respuesta para dosis equimoleculares frente a tamoxifeno, que frente a camptotecina o hidroxiurea.

\section{Conclusiones}

Los cultivos primarios son un excelente primer banco de pruebas para conocer la sensibilidad de un tumor cerebral a un nuevo agente QT o a una nueva indicación de uno ya conocido. Además del tipo de respuesta, dada por la morfología de la curva obtenida, nos aporta una idea de la concentración media, dada por la IC50, para su posterior cálculo en las experiencias in vivo previas a su aplicación clínica. 


\section{Agradecimientos}

A los miembros del Servicio de Neurocirugía del Hospital Universitario Puerta del Mar.

Este trabajo ha sido financiado en parte por la ayuda concedida por el Servicio Andaluz de Salud al grupo de Neuro-oncologia CTS-445.

\section{Bibliografía}

1. Ali-Osmann, F.: Culture of human normal brain and malignant brain tumors for cellular, molecular, and pharmacological studies. En: Human Cells Culture Protocols, edit. Jones, G.E. Human Press, Totowa, New Jersey. 1996.

2. Bredel, M.: Anticancer drug resistance in primary human brain tumors. Brain Res. Rev. 2001; 35: 161-204.

3. Bredel, M., Pollack, I.F., Freund, J.M. et al.: Inhibition of Ras and related G-proteins as a therapeutic strategy for blocking malignant glioma growth. Neurosurgery, 1998; 43: 124-132.

4. Brem, H., Piantadosi, S., Burger, P.C.: Intraoperative controlled delivery of chemotherapy by biodegradable polymers: safety and effectiveness for recurrent gliomas evaluated by a prospective, multi-institutional placebo-controlled clinical triad. Lancet 1995; 345: 1008-1012.

5. Cairncross, J.G., Ueki, K., Zlatescu, M., et al: Specific genetics predictors of chemotherapeutic response and survival in patients with anaplastic oligodendrogliomas. Current treatment of oligodendrogliomas. J. of Natl. Cancer Institut. 1998; 90: 1473-1479.

6. De Bouard, S., Christov, Ch., Guillamo, J,S,, et al.: Invasion of human glioma biopsy specimens in cultures of rodent brain slices: a quantitative analysis. J. Neurosurg. 2002; 97: 169-176.

7. Engebraaten, O., Hjortland, G.O., Hirschberg, H., et al: Growth of precultured human glioma specimens in nude rat brain. J. Neurosurg. 1999; 90: 125-132.

8. Escalona Zapata, J., Casas Huerta, M.J., Diez Nau, M.D., et al.: Estudio del oligodendroglioma y sus variantes por medio de cultivo de tejidos. Rev. Esp. Patol. 2004; 37: 65-72.

9. Gil-Salú, J.L., González-Darder, JM.: Consideraciones sobre la citogenética y los factores de proliferación celular en la oncogénesis de los astrocitomas anaplásicos y glioblastoma multiforme. Neurocirugía, 1998; 9: 199-208.

10. Gil-Salú, J.L., Domínguez-Pascual, I., PérezRequena, J., et al.: Características citomorfológicas e inmunohistoquímicas de cultivos primarios obtenidos a partir de tumores cerebrales. Rev. Esp. Patol. 2002; 35: 207-212.

11. Hegedus, B., Czirók, A., Fazekas, I., et al.: Locomotion and proliferaction of glioblastoma cells in vitro: statistical evaluation of video microscopic observations. J. Neurosurg. 2000; 92: 428-434.

12. Kimmel, D.W., Shapiro, J.R., and Shapiro, W.R.: In vitro drug sensitivity testing in human gliomas. J. Neurosurg.
1987; 66: 161-171.

13. MacDonald, D.R., et al.: Response criteria for phase II studies of supratentorial malignant glioma. Journal of Clinical Oncology. 1990; 8: 1277-1280.

14. Miebach, S., Grau, S., Hummel, V., Rieckmann, P., Tonn, J.C., et al.: Isolation and cultura of microvascular endotelial cells from gliomas of different WHO grades. J Neurooncology. 2006; 76: 39-48.

15. Murphy, K.G., Hatton, J.D., and Sang, H.: Role of glial fibrillary acidic protein expression in the biology of human glioblastoma U-373MG cells. J Neurosurg. 1998; 89: 997-1006.

16. Salcman, M.: Experimental therapy for brain tumors. En Kaye, A.H. and Laws, E.R. (ed). Brain Tumors. London. Churchill\&Livingstone, 2001; pp. 401-420.

17. Schrell, U.M., Rittig, M.G., Anders, M., Kiesewetter F., Marschalek, R., et al.: Hydroxiurea for treatment of unresectable and recurrent meningiomas; part I: Inhibition of primary human meningioma cells in culture and in meningioma trasplants by induction of the apoptotic pathway". J Neurosurg. 1997; 86: 845-852.

18. Schrell, U.M., Rittig, M.G., Anders, M., Koch, U.H., Marschalek, R., et al.: Hydroxiurea for treatment of unresectable and recurrent meningiomas; part II: decrease in the size of meningiomas in patients treated with hydroxyurea. J Neurosurg. 1997; 86: 840-844.

19. Tews, D.S., Nissen, A., Kulgen, C., et al.: Drug resistance-associated factors in primary and secondary glioblastomas and their precursor tumors. J. Neurooncology. 2000; 50: 227-237.

20. Vogel, T.W., Zhuang, Z., Li, J., Okamoto, H., Furuta, M., et al.: Proteins and protein pattern differences between glioma cell line and glioblastoma multiforme. Clin. Cancer Res. 2005; 11: 3624-3632.

21. Westphal, M., Nausch, H., Zirkel, D.: Cell culture of human brain tumors on extracellular matrices. En: Human Cells Culture Protocols, edit. Jones, G.E. Human Press, Totowa, New Jersey. 1996.

22. Yoshizato, K., Zapf, S., Westphal, M., et al.: Thromboxane synthase inhibitors induce apoptosis in migrationarrested glioma cells. Neurosurgery 2002; 50: 343-348.

23. Yuki, K., Uozumi, T., Kodama, Y., et al.: In vitro chemosensitivity test of human brain tumors using a threedimensional organ culture with a collagen gel matrix. J. Neurooncology 1994; 21: 225-232.

Gil-Salú, J.L.; Bosco-López, J.; Domínguez- Villar, M.; Domínguez-Pascual, I.; Pérez-Requena, J.; Palomo, M.J.; López-Escobar, M.: Ensayos de quimiosensibilidad en cultivos primarios de tumores cerebrales. Neurocirugía 2008; 19: 5-11

Correspondencia postal: J.L. Gil Salú; Apartado correos 167; 11100. San Fernando. Cadiz. 
Comentario al articulo: Ensayos de quimiosensibilidad en cultivos primarios de tumores cerebrales, de Gil-Salú y cols.

En este artículo, Gil-Salú y cols. llaman la atención sobre la utilidad del test de MU sobre cultivos primarios de tumores cerebrales, para establecer la quimiosensibilidad de las células tumorales frente a diferentes drogas. Desde su descripción por Mosmann y cols. ${ }^{3}$, en 1983, este test se utiliza en numerosos laboratorios para estudios de quimiosensibilidad tumoral, y en el caso concreto de los gliomas, tanto éste, como otros tests de quimiosensibilidad, pueden predecir, al menos en un $50-70 \%$ de los casos, la respuesta in vivo a la quimioterapia y sobre todo, parecen predecir con alta fiabilidad los casos de quimiorresistencia ${ }^{2}$. No obstante, independientemente de la indudable utilidad de estas técnicas, debemos tener en cuenta que en los tumores cerebrales existen múltiples factores inherentes al huésped, como puede ser la influencia del sistema inmune y su mediación en fenómenos de apoptosis, que pueden condicionar que no haya una estricta correlación entre los datos aportados por los tests de quimiosensibilidad in vitro y la respuesta, in vivo, a un determinado agente quimioterápico ${ }^{4.5 .6 .8}$.

De especial interés, en cuanto a nuevas técnicas capaces de predecir la quimiosensibilidad en tumores cerebrales humanos, es la reciente identificación de marcadores proteicos, que pueden ser detectados con técnicas inmunohistoquímicas y espectrométricas en muestras del tumor, y que parecen ser útiles a la hora de predecir la mayor o menor quimiorresistencia de un determinado glioma maligno cerebral.

\section{Bibliografía}

1. lwadate, Y., Sakaida, T., Saegusa, T., Hiwasa, T., Takiguchi, M., Fujimoto, S., Yamaura, A.: Proteoma-based identification of molecular markers predicting chemosensitivity to each category of anticancer agents in human gliomas. Int. J. Oncol. 2005; 26: 993-998.

2. Kimmel, D.W., Shapiro, J.R., Shapiro, W.R.: In vitro drug sensitivity testing in human gliomas. J Neurosurg. 1987; 66: 161-171.

3. Mosmann, T.: Rapid colorimetric assay for cellular growth and survival: application to proliferation and cytotoxicity assays. J. Immunol. Meth. 1983; 65: 55-63.

4. Nikkhah, G., Tonn, J.C., Hoffmann, O., Kraemer, H.P., Darling, J.L., Schonmayr, R., Schachenmayr W.: The MU assay for chemosensitivity testing of human tumors of the central nervous system. Part I: Evaluation of test-specific variables. J. Neurooncol. 1992; 13: 1-11

5. Nikkhah, G., Tonn, J.C., Hoffmann, O., Kraemer, H.P., Darling, J.L., Schachenmayr W., Schonmayr, R.: The MU assay for chemosensitivity testing of human tumors of the central nervous system. Part II: Evaluation of patient-and drug-specific variables. J. Neurooncol. 1992; 13: 13-24.

6. Puchner, M.J., Giese, A., Zapf, S., Grebe, M., Westphal, M.: Tamoxifen sensitivity-testing of glioblastomas: comparison of in vitro and in vivo results. Acta Neurochir (Wien) 2001; 143: 563-573.

7. Tallen, G., Riabowol, K., Wolff, J.E.: Expression of p33ING1 mRNA and chemosensitivity in brain tumor cells. Anticancer Res. 2003; 23 (2B): 1631-1635.

8. Yung, W.K.: In vitro chemosensitivity testing and its clinical application in human gliomas. Neurosurg. Rev. 1989; 12: 197-203. 